\title{
Distortions of distance and shape are not produced by a single continuous transformation of reach space
}

\author{
GEOFFREY P. BINGHAM \\ Indiana University, Bloomington, Indiana \\ and \\ JAMES A. CROWELL and JAMES T. TODD \\ Ohio State University, Columbus, Ohio
}

\begin{abstract}
We investigated whether distortions of perceived distance and shape could be captured by a single continuous one-to-one transformation of the underlying space. In Experiment 1, the participants reached to touch points around the perimeter of spherical targets viewed at five different distances, to yield simultaneous measures of perceived distance and shape. Different participants reached while using dynamic monocular, static binocular, or dynamic binocular vision. Thin plate spline (TPS) analysis was applied so as to transform a Cartesian grid in such a way as to carry the original target points to the mean reach locations. In all cases, discontinuities appeared in the transformed grid from folding of the space. In Experiment 2, the participants reached to points that lay at the same locus in reach space, but on different portions of the visible target spheres (e.g., front vs. side). The participants reached to different locations when the points were different with respect to shape (e.g., front vs. side) but reached to the same locations when the points were the same with respect to shape (left vs. right side). TPS analysis revealed discontinuities from holes torn in the underlying space. The results show that perceived distance and perceived shape entail different distortions and cannot be captured by a single continuous transformation of reach space.
\end{abstract}

As has been reviewed by Todd, Tittle, and Norman (1995), a large number of space perception studies have shown distortions in the perception of object distance and shape. Distances tend to be overestimated in near space and underestimated in far space (see, e.g., Baird \& Biersdorf, 1967; Ferris, 1972; Gilinsky, 1951). Shapes tend to be expanded in depth, especially within near space, as has been shown both in structure-from-motion (SFM) studies of monocular vision (e.g., Norman \& Todd, 1993; Tittle, Todd, Perotti, \& Norman, 1995; Todd \& Bressan, 1990; Todd \& Norman, 1991) and in studies of stereopsis and binocular vision (e.g., Johnston, 1991; Tittle et al., 1995). Because different results can be obtained when perception is evaluated using passive judgments versus action measures (e.g., Pagano \& Bingham, 1998; see Norman, 2002, for a review), Bingham, Zaal, Robin, and Shull (2000) investigated whether such distortions would be found when reaching was used as a measure of

This work was supported by Grant R01 EY11741-01 A1 from the National Eye Institute to the first author. Correspondence concerning this article should be addressed to G. P. Bingham, Department of Psychology, Indiana University, 1101 East Tenth Street, Bloomington, IN 47405-7007 (e-mail: gbingham @indiana.edu).

Note-This article was accepted by the previous editorial team, headed by Neil Macmillan. perceived distance and shape in near space. They found that both distance and shape were distorted. On average, distances were overestimated, and shapes were expanded in depth, although there were some individual differences, especially in shape perception. These results were confirmed by Bingham, Bradley, Bailey, and Vinner (2001), who compared performances in actual and virtual environments and found comparable results in the two cases. The average shape result, however, was compression in depth. Most recently, Bingham (2003) tested distance, size, and shape perception by measuring reaches to objects at five different distances spanning reach space. Reaches were tested both without and, then, with visual feedback. In the condition without feedback, the participants were able to see the targets both before and during the reach, but they could not see their hands. In the condition with feedback, the participants were able to see their hands together with the targets after each reach had been completed. Static binocular and dynamic monocular and binocular vision were tested with separate groups of participants. The results revealed fairly accurate perception of object distances when the participants used dynamic binocular vision; however, shape was distorted in all the conditions, even with feedback. On average, shapes were reported to be compressed in depth. Overall, the results in that study suggested that distance perception, size perception, and shape perception vary independently. 
We now will investigate whether distortions shown in position perception and in shape perception are produced by a single transformation of reach space. (Note that perception of position entails perception of both egocentric distance and direction.) There are reasons to expect that distortions of perceived position and shape might be the same. The reaching task that we have used and will use is essentially a positioning task. The participants are instructed to reach with a hand-held stylus to the positions of the front, back, left, or right sides of spherical target objects located at different distances. We tested reaches to the back of target objects because typical grasping entails placement of the fingers on the back of an object and the thumb on the front. (Try reaching for your coffee cup on your desk.) The question is, how does one know where the position of the back of an object is. Clearly, shape (and size) information projected from the visible front of an object must be used together with information about the position of the object to determine the position of the back of the object. Thus, information about both shape and position is required to specify the positions of some points. For this reason, perceived position and shape should be expected to interact. Bingham (2003) investigated two different measures of shape, one using reaches to the (occluded) back and one using reaches to the (visible) sides, and found that both yielded the same distortions. The implication was that perceived shape and position interacted strongly. The interaction might ultimately yield a single, uniform, smooth distortion of the space.

However, there are also reasons to expect that perceived shape and position will reflect different distortions, and not a single uniform distortion, of perceived space. There is good evidence that perceived shape cannot be reduced to a perceived set of positions (e.g., Domini \& Caudek, 1999; Todd \& Perotti, 1999). Rather, the evidence shows that observers use information about continuous variations in surface conformation and orientations. Furthermore, aspects of shape (e.g., elliptical vs. cylindrical form) are well detected under conditions in which positions relative to the observer are not well specified-that is, orthographic SFM displays. Perceived position entails specification of direction and egocentric distance. An orthographic display would yield visual directions but not absolute egocentric distances. The latter requires perspective-dependent information. Thus, it is possible that perception of the distances (and thus, positions) of isolated visible points in empty space might yield one set of distortions, whereas the perception of the shapes of extended object surfaces in space might yield a different set of distortions. For instance, perceived distances might be compressed, whereas perceived shapes might be expanded in depth. If perceived shape and perceived position interact to determine perceived positions on object surfaces, the result would be local, inhomogeneous distortions of the space. The global effect of the local distortions would be discontinuities in the space-that is, the space would be folded or torn. We investigated these possibilities in the following experiments.

\section{EXPERIMENT 1}

As is shown in Figure 1, all the participants reached so as to place the midpoint of a hand-held stylus tangent to virtual target spheres - to the front, back, left, or right side of each sphere. ${ }^{1}$ As is shown in the third panel of Figure 1, target spheres were tested at five distances, yielding 20 overlapping locations in reach space, specified in terms of perceived target positions and shapes. Only a single target object was seen at a time, as is shown in the first two panels of Figure 1.

We tested six different viewing conditions. First, the participants used dynamic monocular, dynamic binocular, or static binocular vision to view the target spheres. A different group of participants performed in each condition. The participants using dynamic vision moved their heads from side to side two or three times while viewing the target before each reach. The participants using static vision rested their heads on a chinrest. The participants first performed in a no-feedback session, in which they were allowed to see only a virtual target both before and during the reach. They were not allowed to see the stylus or their hands. In a second, feedback session, the participants were allowed to see a virtual stylus together with the virtual target immediately after each reach had been recorded. To assess their error, they were allowed to move the stylus to position it accurately at the target.

A virtual environment lab was used to test perception and reaching in these experiments. As is shown in Figure 1, the participants wore a head-mounted display (HMD) in which they viewed both the target spheres and, in feedback conditions, a virtual stylus that was coincident with the actual stylus that they held in their hands. Bingham, Bradley, et al. (2001) have measured and described the properties of this lab in detail and have investigated performance in this task in an actual environment, as compared with this virtual environment. They found that performance was comparable. Bingham (2003) has analyzed the reach distances produced by the participants in this experiment and has compared the results with those in other studies with comparable actual environment conditions. The finding was that the results were the same.

\section{Method}

Participants. A total of 22 adults, 19-30 years of age, participated. A different group of participants performed in each visual condition. Nine adults participated in the dynamic monocular condition. Six were male, and 3 were female. Six participated in the dynamic binocular condition. Four were male, and 2 were female. Seven participated in the static binocular condition. Four were male, and 3 were female. The participants were paid $\$ 5 / \mathrm{h}$. All the participants had normal or corrected-to-normal vision (using contacts) and normal motor abilities. All were right-handed.

Apparatus. The virtual environment lab consisted of an SGI Octane graphics computer, a Flock of Birds (FOB) motion measurement system with two markers (for head and hand), and a Virtual Research V6 stereo HMD. Displays in the HMD portrayed a virtual target sphere and a hand-held stylus. The FOB emitter yielded a measurement volume with a $122-\mathrm{cm}$ radius. The emitter was posi- 

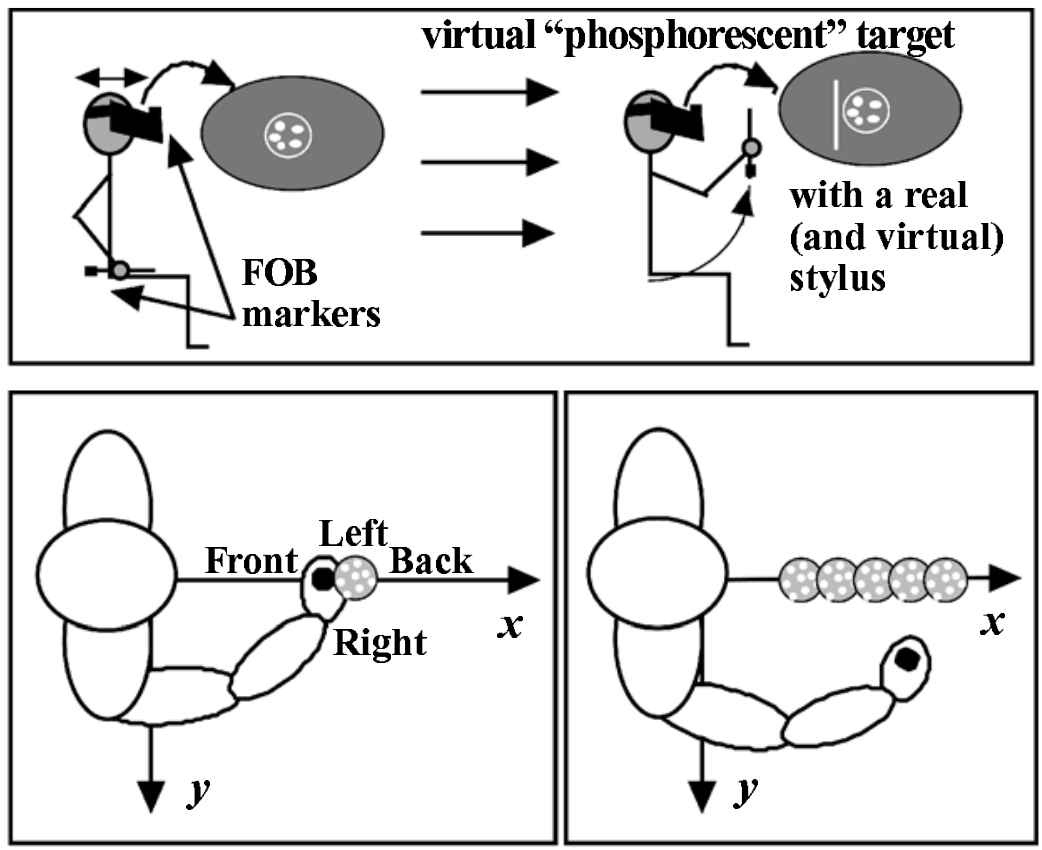

Figure 1. Illustration of the reaching task performed in Experiments 1 and 2. The top panel shows the task. The participant wears a head-mounted display and views a virtual target sphere while holding the stylus in his or her lap and moving his or her head from side to side. Then the participant reaches to touch the sphere with the stylus. A virtual stylus is seen in some conditions after the reach is measured. The second panel shows that the participants reach along the $x$-axis to place a stylus held vertically in the hand tangent to the equator of a target sphere, at its front, back, left, or right side. The $\boldsymbol{x}$-axis is in the depth direction, whereas the $\boldsymbol{y}$-axis is in the frontoparallel direction. The third panel shows the layout of target objects. Only a single target was seen during each trial.

tioned at a height of $20 \mathrm{~cm}$ above the head of the seated participant and at a horizontal distance midway between the head and the hand held at maximum reach. One marker was placed on the V6 HMD, and the other was placed on a Plexiglas stylus held in the participant's hand. The stylus was a Lucite dowel $18.5 \mathrm{~cm}$ in length and $1 \mathrm{~cm}$ in diameter. The 7-cm-diameter virtual target sphere was dark with green phosphorescent-like dots and appeared against a dark background, so that only the green dots could be seen. The stylus and marker was modeled precisely and appeared as a gray virtual stylus with a blue and red marker at its bottom. The hand was not modeled, so that the participants saw only the virtual stylus floating in the dark space. Its position and motion was the same as the actual stylus. There were no shadows cast on the target by the stylus or by the target on the stylus.

The HMD displays subtended a $60^{\circ}$ field diagonally, with complete overlap of the left and the right fields. The resolution was $640 \times 480$ pixels, and the frame rate was $60 \mathrm{~Hz}$. The weight of the helmet was $0.82 \mathrm{~kg}$. The sampling rate of the FOB was $120 \mathrm{~Hz}$. As has been described in Bingham, Bradley, et al. (2001), we measured the the focal distance to the virtual image, the image distortion, the phase lag, and the spatial calibration. The virtual image was at 1-m distance from the eyes. The phase lag was $80 \mathrm{msec}$. The spatial calibration yielded a resolution of about $2 \mathrm{~mm}$.

Procedure. The participant sat in a wooden chair. The experimenter first measured the participant's interpupillary distance, using a ruler, and entered the value into the software. The participant then placed the HMD on his or her head and, following instructions from the experimenter, adjusted the lenses in front of his or her eyes. The participant was allowed a few minutes to move his or her head and hand and to explore and acclimate to the virtual environment. Following this, the maximum reach distance and eyeheight were measured by having the participant hold the stylus out as far as possible in front of his or her face while sitting in the chair and wearing the HMD. The software used the measured values to position the $7-\mathrm{cm}$ virtual sphere at eye height and at distances equal to $.50, .60, .70, .80$, and .90 of the maximum reach.

The task was explained to the participant. The participant was instructed to reach to place the stylus at one of four locations relative to the surface of the target sphere, as is shown in Figure 1. Holding the stylus vertical, he or she reached to place the midpoint of the stylus tangent to the surface of the sphere at its horizontal equator, to the front, right, left, or back. Only the virtual target sphere could be seen, not the virtual stylus, except at the very end of trials in the feedback conditions, at which point the virtual stylus was made visible, as will be explained below.

Between trials, the participants sat holding the stylus in his or her lap. At the beginning of each trial, the target appeared at a given distance, and the computer announced to the participant the location to be touched on the target (e.g., front, back, left, or right). The participant then moved his or her head and torso $10 \mathrm{~cm}$ side to side two to three times at preferred rates while counterrotating the head to keep the target centered in the display and to look at the targeted locus on the surface. The participant then reached at preferred rates. Once the participant had reached the target, he or she said "O.K.," and the three-dimensional (3-D) coordinates of the stylus were recorded. In the conditions without feedback, the participant then placed the stylus back in his or her lap, and the next trial was begun. In the conditions with feedback, the virtual stylus would become 
visible (seen together with the target sphere) at the same time that the 3-D coordinates of the stylus were recorded. The participant was allowed to move the stylus to the correct position on the target if its position was incorrect when the stylus was made visible. Once the participant had done this (which took about $5 \mathrm{sec}$ ), he or she placed the stylus back in his or her lap, and the next trial was begun (with the stylus invisible once again).

A block of trials consisted of reaches to each of the 20 locations (i.e., 4 locations on targets at each of 5 distances) in a completely random order. Five blocks of trials were performed in each viewing condition (i.e., the participants performed 100 reaches in each session $=5$ trials $\times 20$ locations). Reaches were tested in six viewing conditions: the dynamic monocular with no feedback, the static binocular with no feedback, the dynamic binocular with no feedback, the dynamic monocular with feedback, the static binocular with feedback, and the dynamic binocular with feedback. The participants tested with monocular viewing wore a patch over the left eye and performed no-feedback and feedback conditions on subsequent days. In the static binocular conditions, the participants rested their heads on a carved wooden chinrest that sat on top of an aluminum rod that extended from an adjustable clamp on the chair positioned between the participants' legs. The rod did not interfere with reaching. The height of the chinrest was adjusted to a comfortable upright seated posture for each participant. The participants in both binocular conditions were tested first without feedback and then with feedback in separate sessions on a single day, with a 10- to 15-min break between sessions, during which the participants removed the HMD and went for a walk around the department.

\section{Results and Discussion}

We computed a mean $x$ and a mean $y$ for reaches to each of the 20 target locations for each participant in each of the six viewing conditions. Then we computed mean $x$ and $y$ for each of the 20 locations, combining data for the participants in each viewing condition. We compared the 20 target positions with the 20 mean reach positions produced by the participants, to determine whether a single continuous transformation of the underlying reach space could capture the relation between the two sets of points. We used a thin plate spline (TPS) developed in the study of morphology in biology (Dryden \& Mardia, 1998). The TPS performs the analysis suggested by D'Arcy Thompson in his highly influential book On Growth and Form (1961). Assuming a two-dimensional space, the TPS begins by laying a Cartesian coordinate grid over an initial configuration of landmarks representing a shape in the space. Next, given a different target configuration of landmarks (i.e., the result of growth or, in our case, the result of perceptually guided reaching), the analysis transforms the space so as to fit the coordinate grid to the new configuration, moving landmarks on the original shape to corresponding landmarks on the new shape and deforming the coordinate. TPS deforms the grid so as to minimize the total bending. The technique is similar to a cubic spline in one dimension. The analysis yields a relative measure of the total distortion in terms of a bending energy. The analysis also partitions the total transformation into uniform affine components and into remaining components that are either nonaffine or nonuniform.

In preparation for analysis of the reaching data, we performed a set of simulations to illustrate both the analysis and the ways that position and shape distortions appear in the context of the analysis when they occur either separately or as products of a single continuous distortion of the underlying space. First, a uniform stretch of the space transformed by a factor of 1.5 is shown in the second panel of Figure 2. The original configuration of the targets is shown in the top panel of Figure 2. Both the shapes and the target distances have been carried by the stretching of the space. Because this is a uniform affine transformation, it is captured entirely by the uniform affine component of the total transformation, as is shown in the third panel. The fourth panel represents the remaining component and is not different from the original configuration in the first panel. Accordingly, the bending energy in this case is 0 . No bending of the grid was required. Affine transformation allows the grid to be stretched, compressed, or sheared.

As is shown in Figure 3, separate transformations of position or shape could occur in a number of ways. The layout of the targets and the targeted positions is illustrated in the top panel of Figure 1. As is shown in the second panel of Figure 3, position could be stretched by a factor of 1.5 with the shape remaining unchanged. The reader may find this a bit odd and confusing. It might help to think in terms of positioning a target object relative to an observer. The observer misestimates the position (e.g., distance) of the object but gets the shape right nevertheless. What is potentially confusing about this example is that the position of the targets changes, but not the relative positions on the objects. However, this is exactly the point - namely, that shape need not reduce to a set of positions. The results of the TPS analysis in this example are shown in Figure 4. The total transformation is shown in the top panel. The discontinuities (i.e., crossing of grid lines) show that this is not a single continuous (and one-to-one) transformation of the space. The space is folded, and points are lost. As is shown in the second and third panels of Figure 4, this transformation has both uniform affine and nonuniform or nonaffine components. The uniform component is an affine stretch similar to that in the previous example. (It corresponds to the results of linear regressions in analyses of distance perception in previous studies; Bingham, 2003.) The remaining component contains the discontinuities. The bending energy for this transformation was 5.36.

The third panel of Figure 3 illustrates compression of position by a factor of 1.5 with no change in shape. The result of TPS analysis is shown in Figure 5. Again, discontinuities appear. The uniform component is an affine compression. The bending energy was 2.38 .

The fourth panel of Figure 3 illustrates expansion of shape by a factor of 1.5 with no change in position. Again, if this seems odd, refer to the comments in the second example. The result of TPS analysis is shown in Figure 6. Once again, there are discontinuities. However, this time, the entire transformation is in the remaining component. There is no uniform change in the space. Points around the centroid of each shape change, but the 


\section{Uniform stretching of the space}
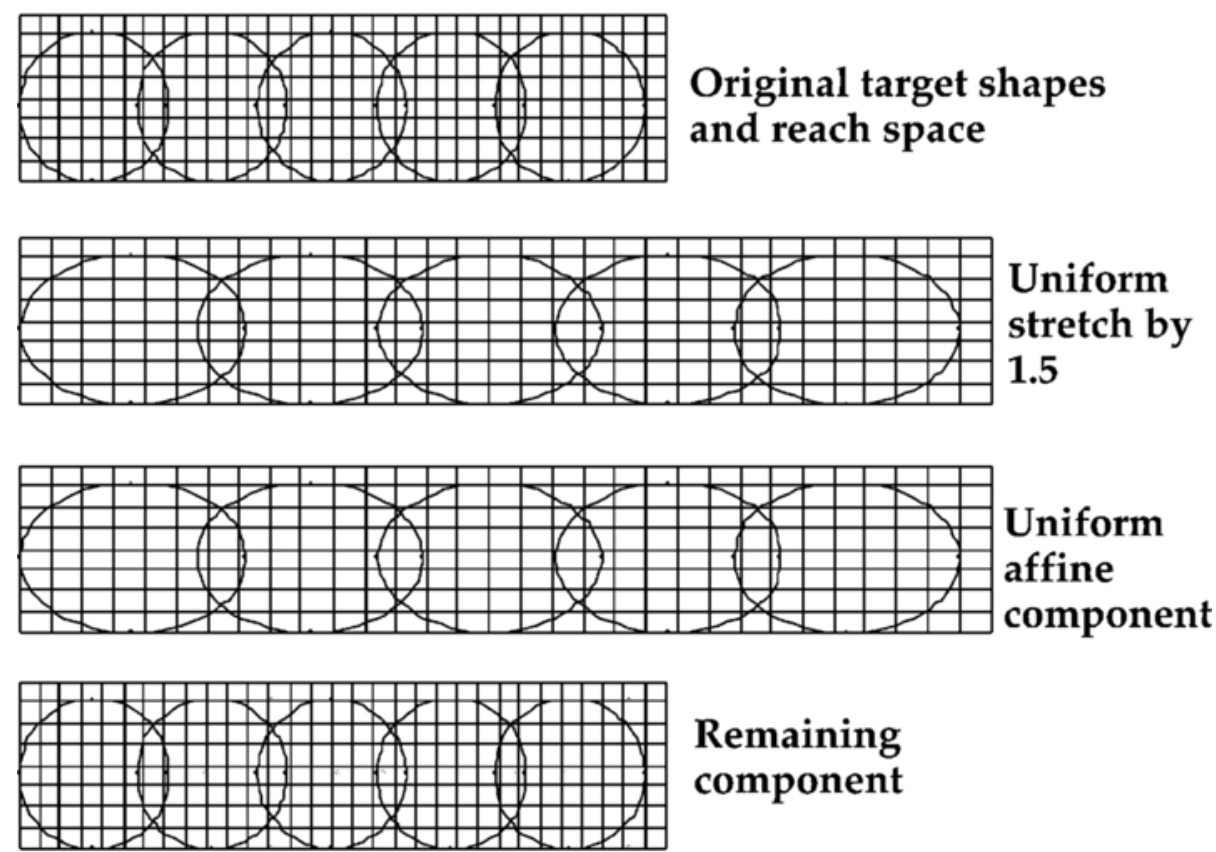

Remaining
component

Figure 2. The effect of a uniform stretching of the space is illustrated. The top panel shows the original set of targets to scale in the context of the Cartesian grid. All transformations illustrated in Figures 2-11 start from this configuration to scale. The second panel shows the total transformation of a uniform stretch by a factor of 1.5 , as fit by a thin plate spline. The third panel shows the uniform affine component of this transformation. The fourth panel shows the remaining component of the transformation.

position of each centroid remains unchanged. (The changes here are affine, but they are nonuniform or local.) The bending energy was 5.36 .

The bottom panel of Figure 3 illustrates compression of shape by a factor of 1.5 with no change in position. The result of TPS analysis is shown in Figure 7. Once again, there are discontinuities, with the entire transformation contained in the remaining component. The bending energy was 2.56 .

So, pure shape changes yield only nonuniform components of a total transformation, whereas pure position changes yield both uniform and nonuniform changes. Compression and expansion yield characteristic patterns, given this particular configuration of landmarks. Finally, independent changes in shape or position - that is, for instance, pure shape change or pure position change-yield discontinuities in the transformed grid, because points in the space are lost and/or gained in the changes.

Finally, in Figure 8, we illustrate a nonlinear transformation of the space. This transformation was designed to be similar to what we eventually saw in the data and to show that a complex change in the space can occur without the appearance of discontinuities. The transformation includes both uniform affine and nonaffine components. We also simulated a strictly nonaffine version of this change (not shown), in which the $d$ and $e$ coefficients in the transformation were set to 1 and 0 , respectively. In ei- ther case, this is a continuous one-to-one transformation of the space. The bending energy was 0.04 . If our results were to look like this, we could conclude that a single continuous (albeit complex) transformation of reach space yielded distortions in the perception of both position and shape. The occurrence of discontinuities in the results would undercut this conclusion.

Next, we performed TPS analyses on the mean reach data, producing the graphs shown in Figures 9-11. To perform this analysis, we used a software package entitled Morphometrika. ${ }^{2}$ We also performed TPS analysis separately on the means for each participant. The TPS analysis uses the mean coordinate values and bends the grid from the original 20 landmarks to fit the 20 mean reach landmarks precisely. The question that remains is how well do the 20 means represent the participant data in each viewing condition. We addressed this question as follows. We regressed the $20 x$ means for a given viewing condition on the set of means for the individual participants in that condition. Of course, the simple linear regression yielded a line with a slope of 1 and an intercept of 0 in each case, so the $r^{2}$ is a direct measure of the percentage of the variance in participant means captured by the respective viewing condition means.

Dynamic monocular vision. Figure 9 shows the results of the TPS analysis in the dynamic monocular conditions. We show the total result and the components for 
Original target shapes and positions

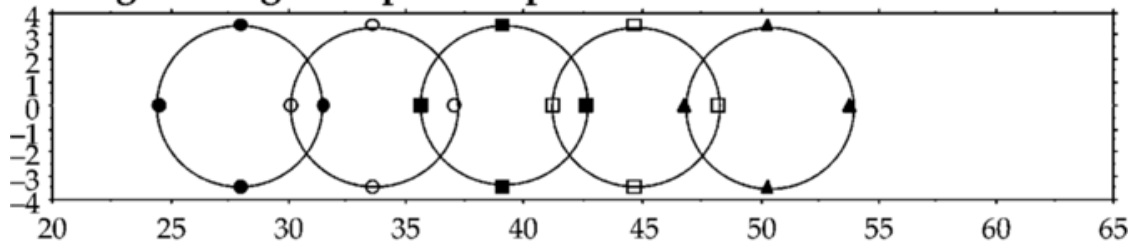

Position expansion or stretch

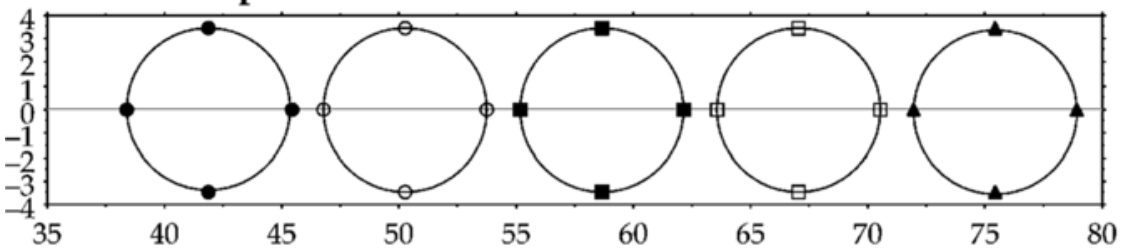

Position compression

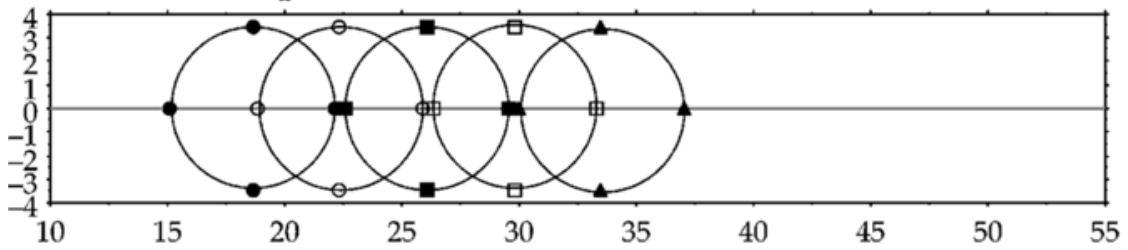

Shape expansion or stretch

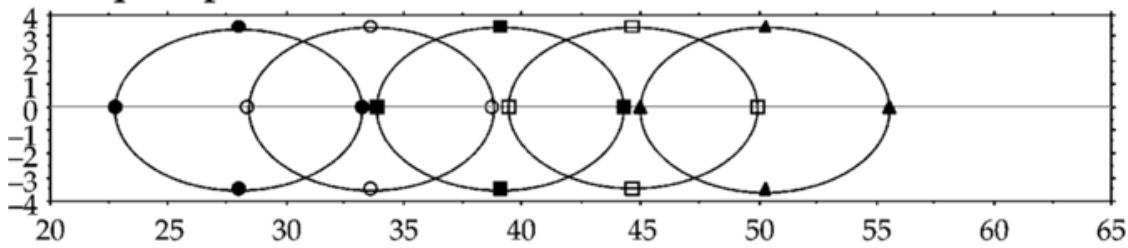

Shape compression

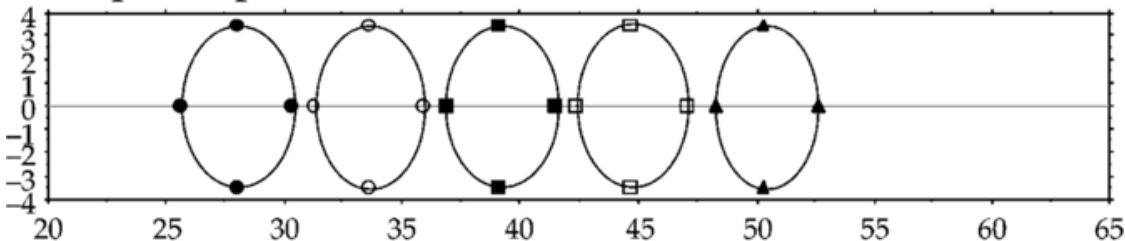

Figure 3. The top panel shows the layout of targeted locations in reach space as four locations on a target sphere located at each of five distances. The $x$ - and $y$-axes show extents in centimeters. The second panel illustrates an expansion of target positions by a factor of 1.5 without a change in target shapes. (Compare Figure 4.) The third panel illustrates compression of target positions by a factor of 1.5 without a change in target shapes. (Compare Figure 5.) The fourth panel illustrates expansion of target shapes by a factor of 1.5 without a change in target positions. (Compare Figure 6.) The fifth panel illustrates compression of target shapes by a factor of 1.5 without change in object positions. (Compare Figure 7.)

the no-feedback condition and just the total result for the feedback condition. (The components look similar to those in the no-feedback condition, except that the amount of compression in the uniform affine component was much less.) Discontinuities were produced in both viewing conditions. Also, the transformation involved both uniform affine and nonuniform or nonaffine components. The affine change was a compression in depth. The bending energy in the no-feedback condition was 7.62. The bending energy in the feedback condition was 2.35.
The regression of the no-feedback condition $x$ means on the participant $x$ means yielded an $r^{2}$ of $.64[F(1,178)=$ $322.9, p<.001]$. For $y$ means, the result was $r^{2}=.61$ $[F(1,178)=273.7, p<.001]$. The results with feedback were, for $x, r^{2}=.87[F(1,178)=1170.2, p<.001]$ and, for $y, r^{2}=.58[F(1,178)=248.5, p<.001]$. It was apparent in the $y$ scatter plots that 1 participant was deviating from the rest to one side. When the regressions were redone without the data from that participant (recomputing the condition means), the $r^{2}$ for $y$ increased to .82 


\section{Position expansion or stretch}
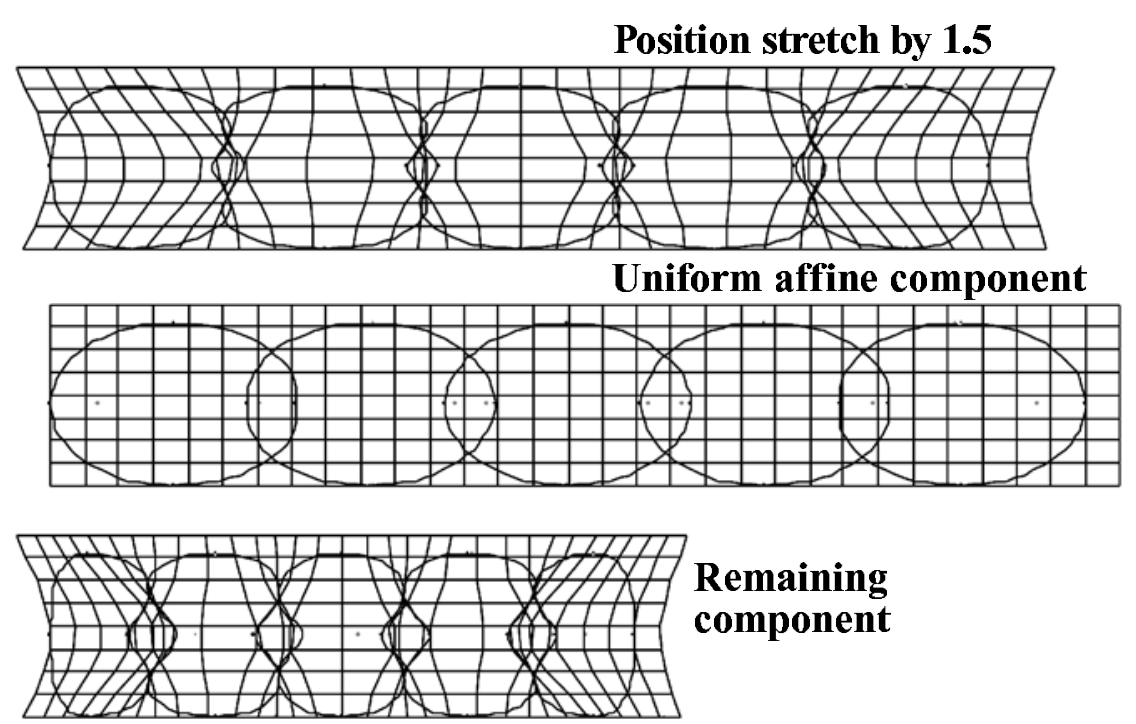

\section{Remaining component}

Figure 4. The effect of an expansion of object position without change in object shape is illustrated. The top panel shows the total transformation of the stretch in position by a factor of 1.5, as fit by a thin plate spline. The second panel shows the uniform affine component of this transformation. The third panel shows the remaining component of the transformation.

without feedback and .85 with feedback. However, when we repeated the TPS analysis with the recomputed means, the results were essentially the same. Without feedback, the bending energy was 10.05 , and with feedback, it was 2.99 . When we did TPS analysis separately for each participant in each condition, all yielded discontinuities and affine compression in depth. The median bending energy without feedback was 8.13 , and with feedback, it was 7.87 . Bending energies decreased for 6 of the 9 participants between the no-feedback and

\section{Position compression}

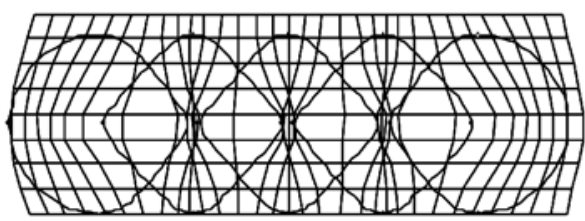

Position compression by 1.5

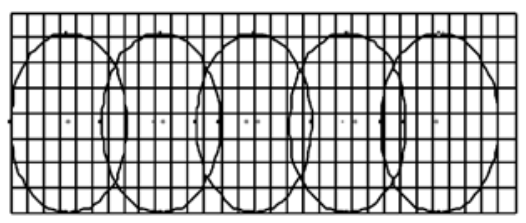

\section{Uniform \\ affine component}

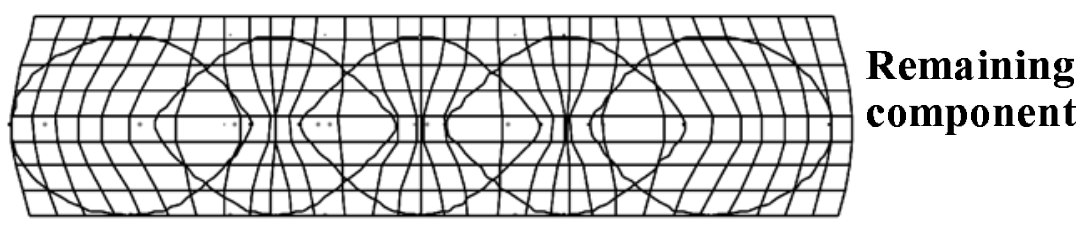

Figure 5. The effect of a compression of object position without change in object shape is illustrated. The top panel shows the total transformation of the compression in position by a factor of 1.5, as fit by a thin plate spline. The second panel shows the uniform affine component of this transformation. The third panel shows the remaining component of the transformation. 
Shape expansion or stretch

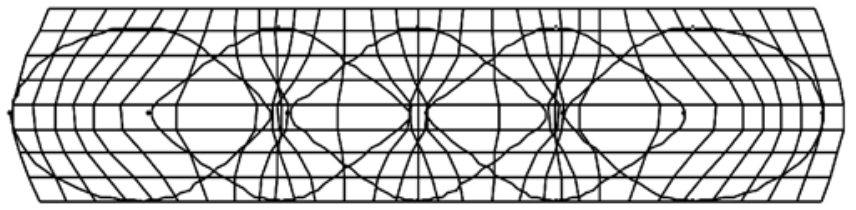

Shape stretch by 1.5

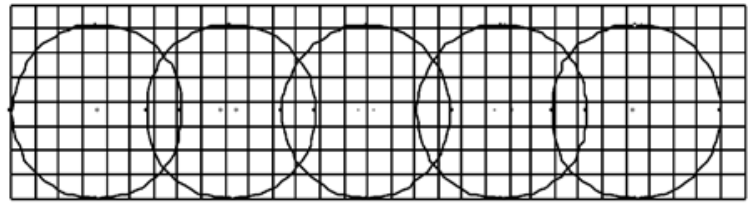

Uniform

affine component

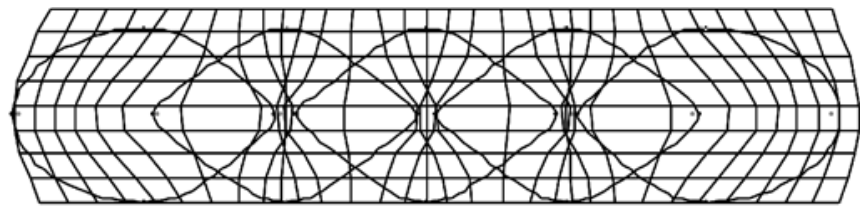

Remaining component

Figure 6. The effect of an expansion of object shape without change in object position is illustrated. The top panel shows the total transformation of the stretch in shape by a factor of 1.5 , as fit by a thin plate spline. The second panel shows the uniform affine component of this transformation. The third panel shows the remaining component of the transformation.

the feedback conditions. The reduction in the amount of compression in the uniform affine component between the feedback conditions was consistent with the finding in Bingham (2003) that the slope of the linear regression of target distances on mean reach distances increased between feedback conditions from slope $=.65$ to .75 .

Static binocular vision. Figure 10 shows the results of the TPS analysis in the static binocular conditions.

\section{Shape compression}

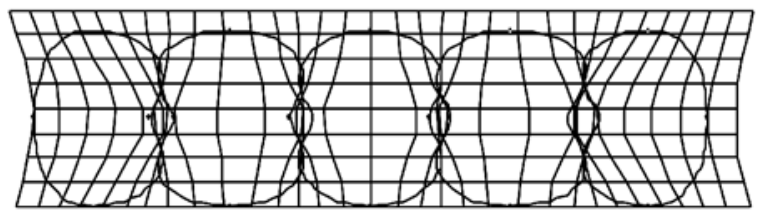

\section{Shape compression by 1.5}

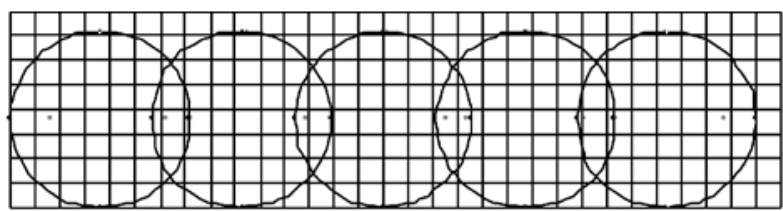

\section{Uniform affine component}

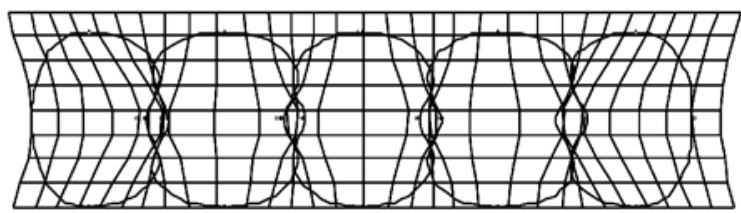

\section{Remaining component}

Figure 7. The effect of a compression of object shape without change in object position is illustrated. The top panel shows the total transformation of the compression in shape by a factor of 1.5, as fit by a thin plate spline. The second panel shows the uniform affine component of this transformation. The third panel shows the remaining component of the transformation. 


\section{Continuous Nonlinear Transformation of Space}

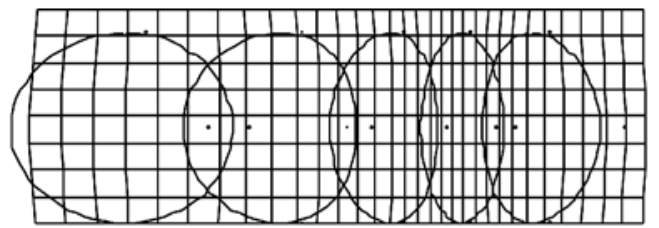

Nonlinear transformation:

$$
x^{\prime}=\underbrace{a \sin [b x-c]}_{\text {Non-affine }}+\underbrace{d x+\mathrm{e}}_{\text {Affine }}
$$

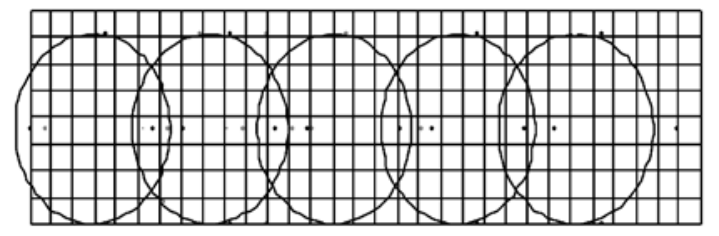

\section{Uniform affine component}

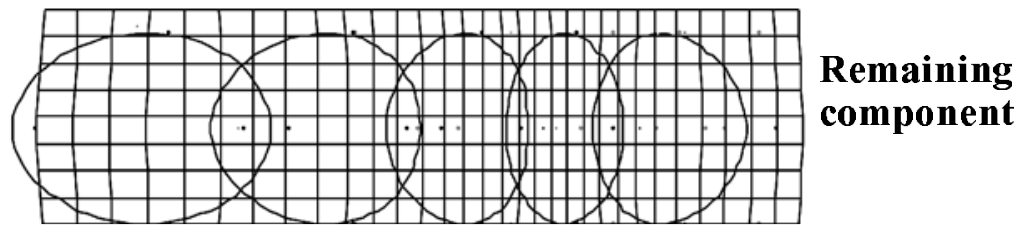

Figure 8. The effect of a single nonlinear transformation of the space is illustrated. The top panel shows the total transformation, as fit by a thin plate spline. The transformation includes both uniform affine and nonaffine components. The second panel shows the uniform affine component of this transformation. The third panel shows the remaining component of the transformation.

Discontinuities were produced in both the no-feedback and the feedback conditions. Again, the uniform affine component was a compression in depth, although the amount of compression was less than that in the dynamic monocular condition. This is consistent with the results of Bingham (2003), who found that linear regression of target distances on reach distances yielded slopes of .84, which was greater than slopes in the dynamic monocular condition (mean slope $=.70$ ). The bending energy in the no-feedback condition was 1.34 . The bending energy in the feedback condition was 1.56 . These were smaller than those in the dynamic monocular condition.

The regression of the no-feedback condition $x$ means on the participant $x$ means yielded an $r^{2}$ of .76 $[F(1,138)=$ $426.3, p<.001]$. For $y$ means, the result was $r^{2}=.45$ $[F(1,138)=113.7, p<.001]$. The results with feedback were, for $x, r^{2}=.88[F(1,138)=1005.7, p<.001]$, and, for $y, r^{2}=.82[F(1,138)=628.1, p<.001]$. Again, when we did TPS analysis separately for each participant in each condition, all yielded discontinuities and affine compression in depth. The median bending energy without feedback was 2.99, and with feedback, it was 5.43. Bending energies increased for 4 of the 7 participants between the no-feedback and the feedback conditions. However, the amount of affine compression was less with feedback.

Dynamic binocular vision. Figure 11 shows the results of the TPS analysis in the dynamic binocular conditions. Discontinuities were produced in both the nofeedback and the feedback conditions, and the uniform affine component was compression in depth. This condition yielded the smallest amount of affine compression, on average, of the three no-feedback viewing conditions. The bending energy in the no-feedback condition was 1.46 . The bending energy in the feedback condition was 5.28.

The regression of the no-feedback condition $x$ means on the participant $x$ means yielded an $r^{2}$ of .78 $[F(1,118)=$ $427.1, p<.001]$. For $y$ means, the result was $r^{2}=.70$ $[F(1,118)=269.9, p<.001]$. The results with feedback were, for $x, r^{2}=.96[F(1,118)=2,575.8, p<.001]$, and, for $y, r^{2}=.89[F(1,118)=994.1, p<.001]$. Again, we did TPS analysis separately for each participant in each condition. For the first time, 2 participants exhibited affine stretch in the no-feedback condition, but only 1 continued to do so in the feedback condition. The remaining participants exhibited affine compression. All the participants exhibited discontinuities in the transformed grids. The median bending energy without feedback was 6.94 , and with feedback, it was 7.66. Bending energies increased for 4 of the 6 participants between feedback conditions. Uniform affine compression decreased between the feedback conditions and was nearly absent in the mean with feedback result.

These results confirm those of Bingham (2003). Although feedback yielded improvements in $r^{2}$ values and reductions in the amount of affine compression (or increases in the slopes of distance functions), it did not yield better performance with respect to shape. In fact, with binocular vision, performance with respect to shape got worse. Also, and more to the point of the present 


\title{
Dynamic Monocular Vision
}

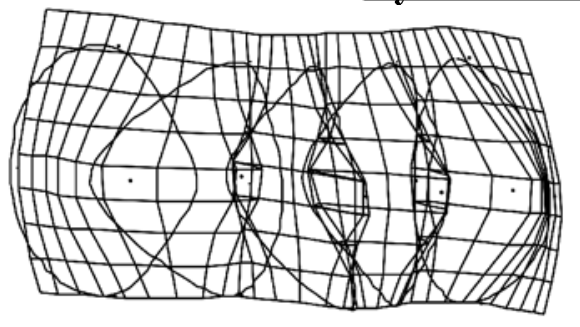

\author{
No Feedback: \\ Total transformation
}
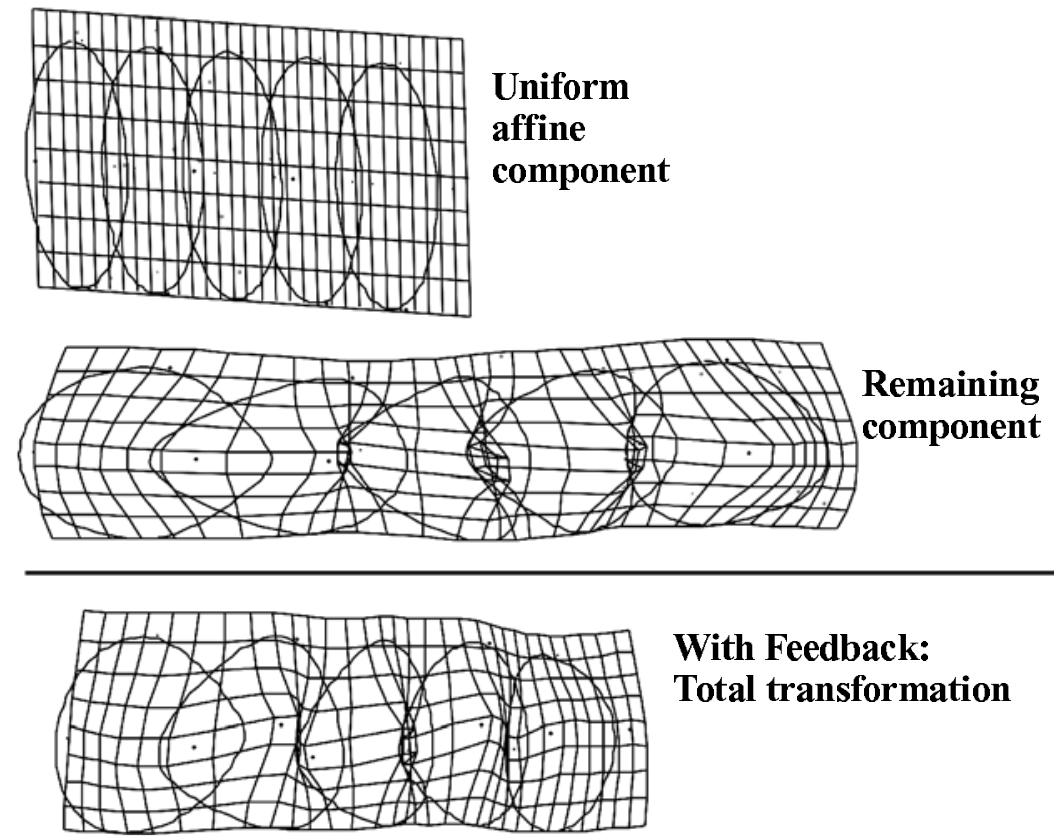

\section{With Feedback: Total transformation}

Figure 9. Results of the thin plate spline analysis applied to the reach means in the dynamic monocular vision condition. The top panel is the total transformation result in the no-feedback condition. The second panel is the uniform affine component of this transformation. The third panel is the remaining component of the transformation. The fourth and last panel is the total transformation result in the with-feedback condition.

question, the discontinuities found as a result of the TPS analyses indicate that the distortions in distance and shape cannot be attributed to a single continuous transformation of reach space. ${ }^{3}$

Looking especially at the TPS results for binocular vision conditions, we found that they were similar to the simulations for compression of position shown in Figure 5. In this case, both uniform affine compression and discontinuities appeared. Also, the shape of the deformed grid was similar to that in the results. Only position changes produce changes in the uniform affine component. Compression of position is consistent with the low slopes in distance functions shown in Bingham (2003). However, pure position change would imply no distortion of shape. The analyses performed in Bingham yielded compression of shape in depth. So, the results must also involve shape distortions. The main finding from the TPS analyses is the discontinuities that imply that distortions of shape and distance (or position) are different. Indeed, Bingham found that shape distortions were not improved by feedback, although accuracy of distance was improved.

\section{EXPERIMENT 2}

The target configuration in Experiment 1 involved object positions that overlapped, so that the same positions in space were occupied (at different times) by different objects. However, because the targeted locations of reaches on the objects were discrete and relatively sparse, the targets on different objects were not located at identical positions in reach space. The strongest test of single versus multiple transformations would be provided by a configuration of targets in which the same position in reach space was occupied (at different times) by different locations on target shapes. This would allow a statistical test of the question. When locations are different in terms of locus within a shape, although identical in reach space position, do reaches to that locus yield a single distribution of reaches or two distributions of 


\section{Static Binocular Vision}

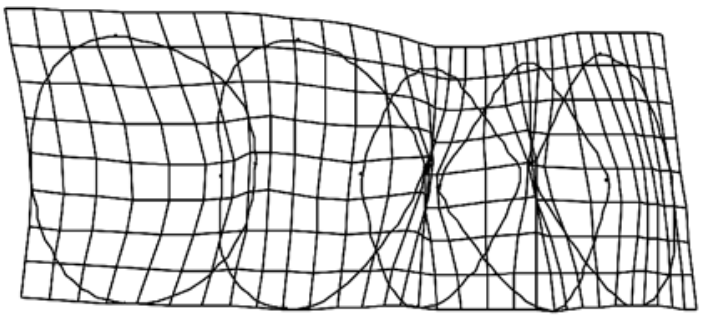

No Feedback:

Total transformation
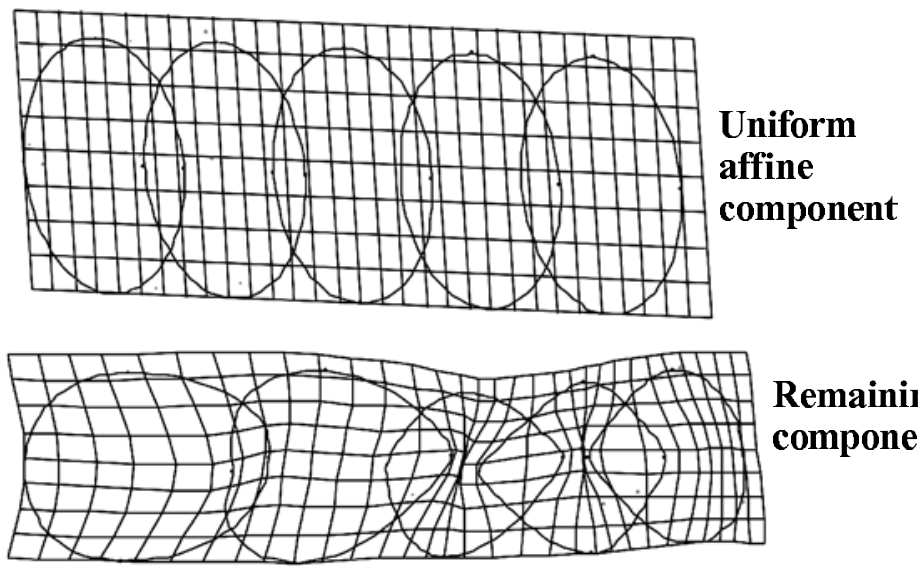

Remaining component

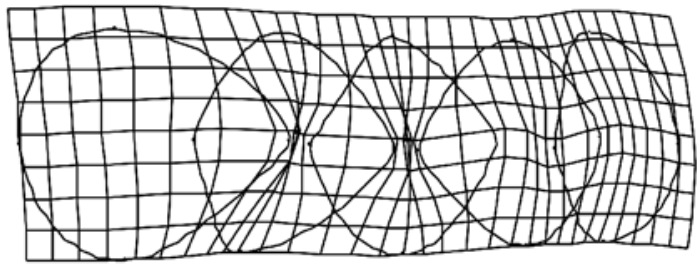

With Feedback: Total transformation

Figure 10. Results of the thin plate spline analysis applied to the reach means in the static binocular vision condition. The top panel is the total transformation result in the no-feedback condition. The second panel is the uniform affine component of this transformation. The third panel is the remaining component of the transformation. The fourth and last panel is the total transformation result in the with-feedback condition.

reaches? Alternatively, when locations are the same in terms of locus within a shape as well as in reach space, do reaches to that locus yield a single distribution in the space? We tested this design, using a configuration of six spherical targets, as is shown in Figure 12.

The configuration is best understood as two overlapping triads of target spheres. In one triad, left and right side points of the nearest target appear at the same position in reach space as the front points on two targets that are farther away and to either side, respectively. In a second triad, the front point of the farther target in the center is at the same position as the side points on two nearer targets. Similar relations occur in terms of coincident back points and side points. In each case, if the effects of shape and of distance are different in perception, reaches to these points, which are positioned identically in reach space but differently in the context of object shapes, should yield differences in reaching.
The configuration of targets also contains locations where points on the sides of different targets appear at the same position in reach space. These points are the same both in terms of position in the space and in the context of object shape, so they should not yield differences in reaching.

Finally, to ensure that the participants could distinguish near and far targets adequately so that they were not merely confusing targets as lying at the same distances, we tested to be sure that reaches to near and far targets were different, as they should have been.

If reaches to front and side points that are coincident in reach space yield differences in reaching, the effect will be to distort reach space perceptually so as to tear a hole in the space (as was mentioned in the introduction). These topological violations (the folding so as to lose points, as was shown in Experiment 1, and tearing so as to gain points, as was predicted here) indicate that dis- 


\section{Dynamic Binocular Vision}

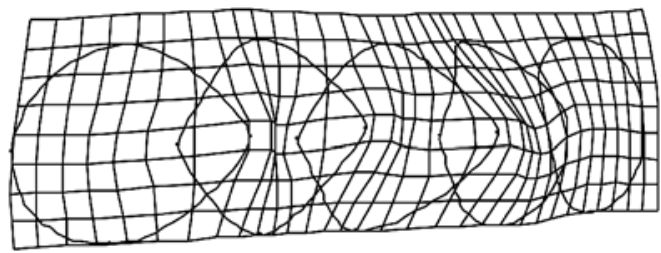

\section{No Feedback: \\ Total transformation}

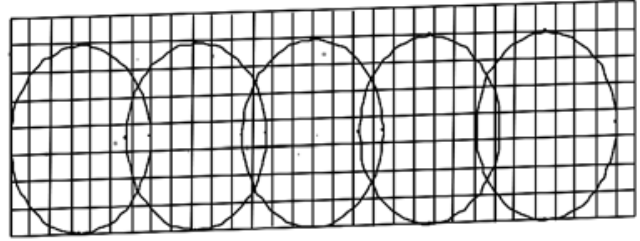

\section{Uniform \\ affine \\ component}

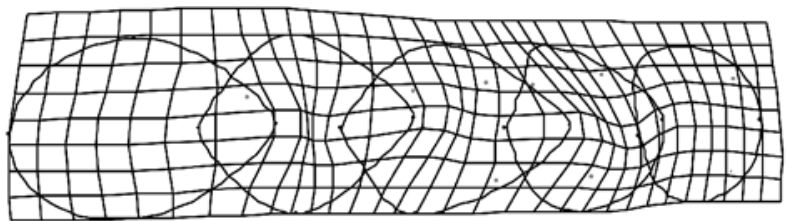

\section{Remaining component}

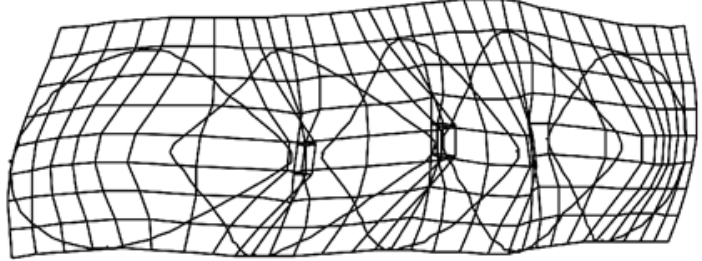

\section{With Feedback: Total transformation}

Figure 11. Results of the thin plate spline analysis applied to the reach means in the dynamic binocular vision condition. The top panel is the total transformation result in the nofeedback condition. The second panel is the uniform affine component of this transformation. The third panel is the remaining component of the transformation. The fourth and last panel is the total transformation result in the with-feedback condition.

tance (or location) perception and shape (or relative distance) perception entail different distortions or transformations.

\section{Method}

Participants. Six adults, 22-46 years of age, participated. One was an author, G.P.B. The rest were graduate students who were naive about the experimental question. Five participants were male, and 1 was female. Graduate student participants were paid $\$ 7 / \mathrm{h}$. All the participants had normal or corrected-to-normal vision (using contacts) and normal motor abilities. All were right-handed.

Procedure. The apparatus and procedure were the same as those in Experiment 1, with the following exceptions. Only dynamic binocular vision was tested without feedback. A configuration of spherical targets, each $7 \mathrm{~cm}$ in diameter, was used, as is shown in Figure 12. The two center targets were in the observer's sagittal plane, and the nearer of these was placed at $70 \%$ of maximum reach. Before being tested, each participant calibrated his or her reaching by performing 12 reaches to this center-near target ( 3 reaches to each of the four locations in a random order), with vision of both the virtual target and the virtual stylus throughout each reach. These reaches were very accurate. Side targets were positioned $3.5 \mathrm{~cm}$ to the left and right of the center targets. Far targets were positioned $3.5 \mathrm{~cm}$ behind near targets. Four locations (front, back, left side, and right side) on six targets yielded 24 positions to be reached. These were visited in a random order in each of four blocks of trials, requiring that a total of 96 reaches be performed without feedback (after calibration trials).

\section{Results and Discussion}

Mean $x$ and $y$ values for each of the 24 locations were computed for each participant. We could not perform a TPS analysis in which the original target points were taken to the reach means, because this involved one-to-many mappings, which caused the analysis to blow up. However, TPS analysis is tolerant of many-to-one mappings, as was demonstrated in Experiment 1 and as has been discussed by Dryden and Mardia (1998, p. 212). So, in these cases, we performed the analysis in the reverse direction, going from the reach mean coordinates to the original target coordinates. The total transformation results for the 6 par- 

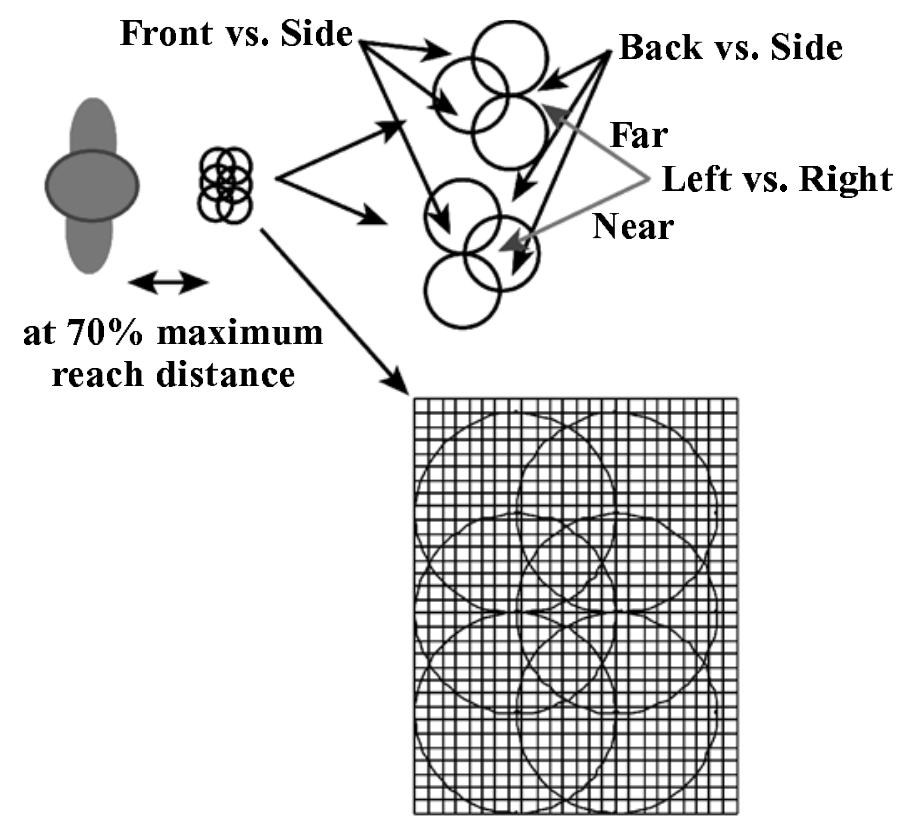

Figure 12. Illustration of the layout and design of targets in Experiment 2 . See the text for details. The target configuration is also shown with the Cartesian grid to scale. The results of the thin plate spline analyses shown in Figure 13 are to scale from this initial configuration.

ticipants are shown in Figure 13. The consistent occurrence of discontinuities from folding is apparent. Each of these implies a tearing of the space when the transformations are performed in the reverse order.

Next, we performed a separate analysis of variance (ANOVA) on the data for each participant, testing the difference of front and side points at the three coincident locations. The results are shown in Table 1. Significant differences were obtained for half the participants. The same analysis was performed for coincident back and side points. The results are shown in Table 2. Significant differences were found for 5 of the 6 participants.

Next, we performed a separate two-factor ANOVA on the data for each participant, testing coincident side points that were located on near and far targets. The two factors were left versus right and near versus far. We expected the former factor not to reach significance, whereas the latter was expected to be significant. The results are shown in Tables 3 and 4 . Left versus right was significant only for 1 of the 6 participants, whereas near versus far was significant for 5 of the 6 . We performed this analysis again using all side points (instead of just the coincident ones). The pattern of results was essentially the same as that shown in Tables 3 and 4 . Left versus right was significantly different for only 2 of 6 participants, whereas near versus far was significant for all the participants.

We computed mean coordinates for the 3 participants who showed significant differences between coincident front and side points. Then we computed means for the 3 remaining participants. The former are plotted in Fig- ure 14 separately for the two target triads. It is apparent that these participants did not substantially distort target positions, but they did distort the shape, expanding shapes in depth. The result was that coincident front and side and coincident back and side points became strongly separated, as is shown in the figure. We subjected these means to a TPS analysis, and the resulting affine component grid is shown in the top panel of Figure 15. The lack of a significant affine distortion is apparent, consistent with no distortion of positions. In Figure 16, we plotted the means for the remaining 3 participants, who failed to show significant differences between front and side points. These participants appear to have kept shape reasonably accurate while distorting position. This is confirmed by the affine component grid shown in the second panel of Figure 15. The TPS was performed in reverse, transforming from reach means to target landmarks. Thus, the affine stretch shown in Figure 15 should be interpreted as compression of positions. So, some participants distorted shape but not position, whereas others distorted position but not shape. Either way, there was not a single continuous (one-to-one) transformation of underlying reach space that was responsible for the distortions that appear in these data.

\section{GENERAL DISCUSSION}

Perceptionists have often treated perceived properties in space perception as if they were part of a single underlying coherent perceptual space. Accordingly, perception- 

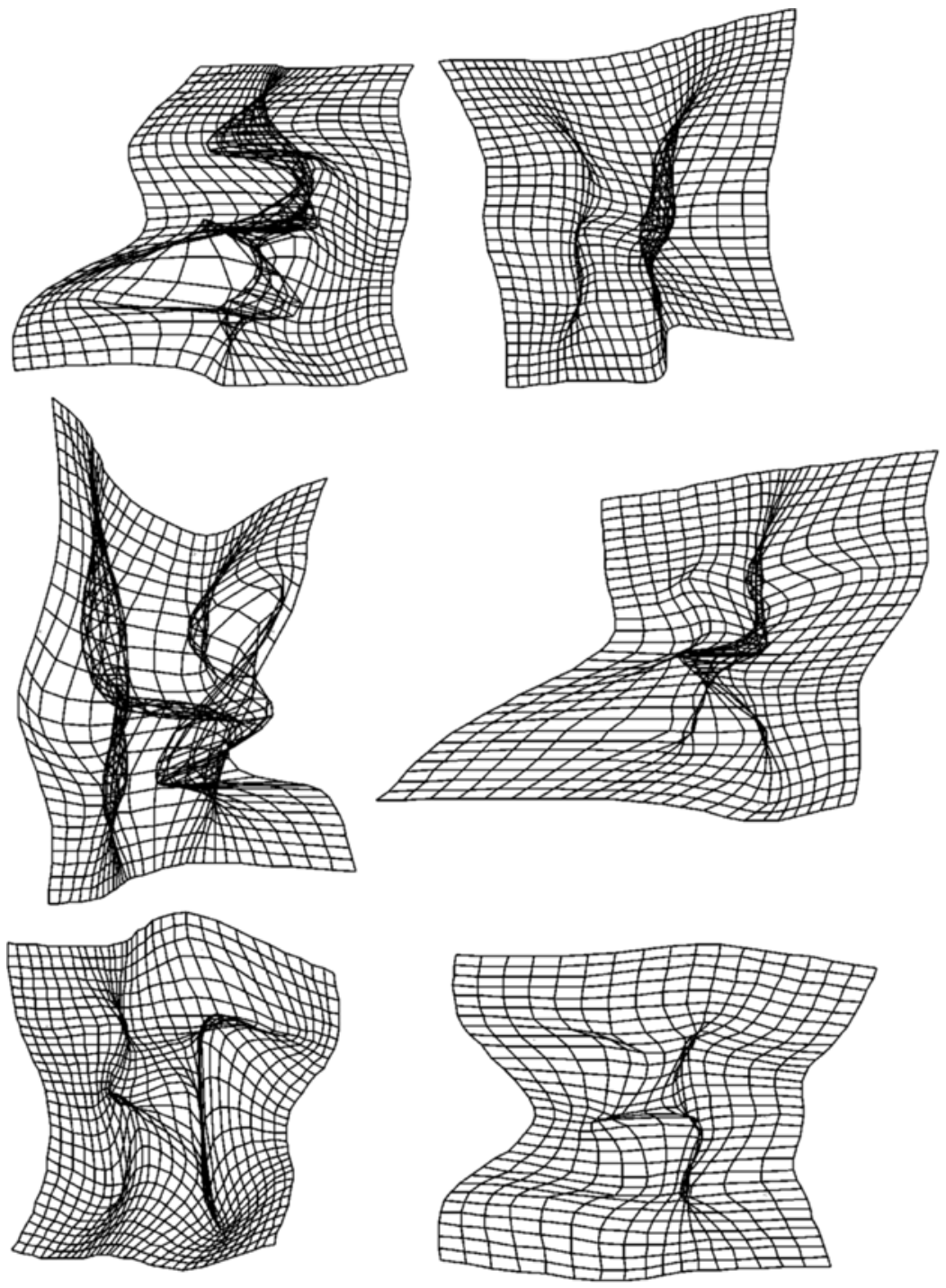

Figure 13. The total transformation results of a thin plate spline analysis performed on the reach means of each of the 6 participants in Experiment 2. Each analysis was performed in reverse, transforming reach means to the original target locations.

ists have debated which single geometry would capture the structure of visual space (e.g., Indow, 1991; Luneburg, 1950; Todd et al., 1995; Wagner, 1985). Related to this, Helmholtz (1925) and others both before and since have assumed that perceived size and perceived distance should covary in a systematic way (e.g., Brenner \& van Damme, 1997, 1999; Gilinsky, 1951; Gogel, 1977; Hochberg, 1978). More recently, perceptionists have assumed that perceived distance and shape should covary in a systematic fashion (Brenner \& van Damme, 1997, 1999; Johnston, 1991). In these cases, it is rather natural to think of each point on the surface of a perceived object shape as being perceived in terms of its respective egocentric distance from the observer, in which case perceived shapes and sizes would indeed covary with per- ceived distance. However, this is not consistent with the understanding of shape perception that has been emerging from studies of both SFM and stereopsis.

Object perception frequently occurs under conditions of small-angle vision. Objects are often viewed in cir-

Table 1

Front Versus Side

\begin{tabular}{crccc}
\hline & & \multicolumn{3}{c}{ Mean } \\
Participant & $F(1,26)=$ & $p$ & Difference & $S D$ \\
\hline P1 & 6.6 & $<.02$ & 1.4 & 1.3 \\
P2 & 18.3 & $<.001$ & 6.7 & 4.1 \\
P3 & 3.0 & n.s. & 0.6 & 2.8 \\
P4 & 3.6 & n.s. & 0.7 & 1.6 \\
P5 & 1.2 & n.s. & 0.6 & 1.4 \\
P6 & 9.2 & $<.01$ & 3.8 & 3.3 \\
\hline
\end{tabular}


Table 2

Back Versus Side

\begin{tabular}{ccccc}
\hline & & \multicolumn{3}{c}{ Mean } \\
Participant & $F(1,26)=$ & $p$ & Difference & $S D$ \\
\hline P1 & 22.0 & $<.001$ & 3.2 & 1.7 \\
P2 & 30.1 & $<.001$ & 6.6 & 3.1 \\
P3 & 3.6 & n.s. & 0.8 & 2.2 \\
P4 & 51.5 & $<.001$ & 5.7 & 1.6 \\
P5 & 78.0 & $<.001$ & 3.4 & 1.4 \\
P6 & 8.5 & $<.01$ & 3.4 & 3.0 \\
\hline
\end{tabular}

Table 3

Left Versus Right

\begin{tabular}{|c|c|c|c|c|c|c|}
\hline Participant & $F(1,12)=$ & $p$ & $\begin{array}{c}\text { Mean } \\
\text { Difference }\end{array}$ & $F(1,44)=$ & $p$ & $\begin{array}{c}\text { Mean } \\
\text { Difference }\end{array}$ \\
\hline P1 & 1.7 & n.s. & 1.2 & 0.7 & n.s. & 0.4 \\
\hline P2 & 3.4 & n.s. & 3.5 & 4.7 & $<.04$ & 1.9 \\
\hline P3 & 0.2 & n.s. & 0.3 & 0.8 & n.s. & 0.8 \\
\hline $\mathrm{P} 4$ & 5.0 & $<.05$ & 2.3 & 25.9 & $<.001$ & 2.4 \\
\hline P5 & 0.1 & n.s. & 0.2 & 1.1 & n.s. & 0.6 \\
\hline P6 & 0.4 & n.s. & 0.9 & 0.3 & n.s. & 0.5 \\
\hline
\end{tabular}

Table 4

Near Versus Far

\begin{tabular}{crccccc}
\hline \multicolumn{7}{c}{ Mean } \\
Participant & $F(1,12)=$ & $p$ & Difference & $F(1,44)=$ & $p$ & $\begin{array}{c}\text { Mean } \\
\text { Difference }\end{array}$ \\
\hline P1 & 17.1 & $<.002$ & 3.6 & 38.5 & $<.001$ & 3.0 \\
P2 & 4.5 & $<.05$ & 4.1 & 16.7 & $<.001$ & 3.8 \\
P3 & 5.9 & $<.04$ & 4.3 & 22.9 & $<.001$ & 4.1 \\
P4 & 0.8 & n.s. & 0.9 & 8.1 & $<.01$ & 5.0 \\
P5 & 20.0 & $<.001$ & 3.0 & 42.6 & $<.001$ & 3.6 \\
P6 & 4.4 & $<.05$ & 3.1 & 31.3 & $<.001$ & 4.8 \\
\hline
\end{tabular}

cumstances in which they subtend angles of about $4^{\circ}-6^{\circ}$ at the eye. This is true, for instance, when you view a coffee cup on a desk at which you are seated or when you look at the face of someone sitting across from you at the dinner table. Visual information for shape perception under such small-angle viewing is appropriately modeled as scaled orthographic projection (e.g., Lind, 1996). Some of the information that would be available in full perspective projection is not available, because it falls below the level of resolution (Howard \& Rogers, 1995; Koenderink \& van Doorn, 1987). Scaled orthographic projection means that the surface structure of an object is projected orthographically or in parallel for purposes of shape perception to an image plane centered in the object. That image, which is at the average distance of the object surface points, is then effectively polar projected to the eye. Scaled orthographic projection allows the average distance of an object to be specified without the distance of each point of the object surface being specified in the same way. Because object surface structure is specified by parallel projection, only affine shape can be perceived (Koenderink \& van Doorn, 1991). Relative depth order along a surface is specified, but not the metric distances between points. Affine means that distances along the depth direction cannot be intrinsically related to distances along the frontoparallel direction. A similar analysis applies to stereopsis, where the perspective structure is absent in vertical disparities, leaving only horizontal disparities (Howard \& Rogers, 1995).

Affine shape predicts that perceived shapes should vary randomly - that is, they could exhibit expansion or compression in depth. Variations of this sort do appear as individual differences in shape perception results. Some observers yield expanded shapes, and others compressed
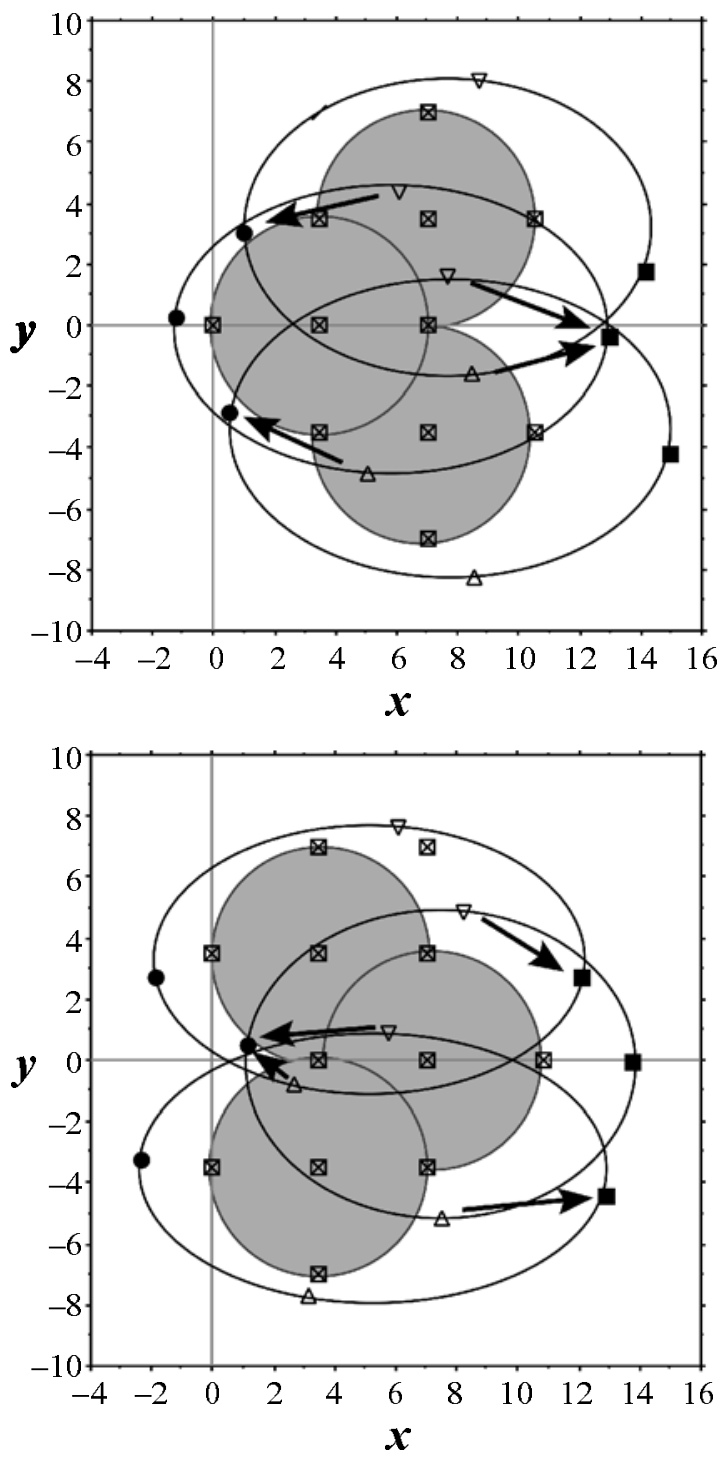

Figure 14. Reach means computed using the data of the 3 participants that yielded significant differences between front and side points. Results are plotted for two triads of targets, one involving coincidence of front and side points and the other involving coincidence of back and side points. The separation of reach means for points that were coincident on target objects is shown by arrows (e.g., side and front points that were coincident in successive target placements but separated in the resulting mean reach locations). Original target points (front, back, left, and right) on each object, cross hatched squares; mean reaches to side points, open inverted triangles; mean reaches to front points, filled circles; mean reaches to back points, filled squares. 

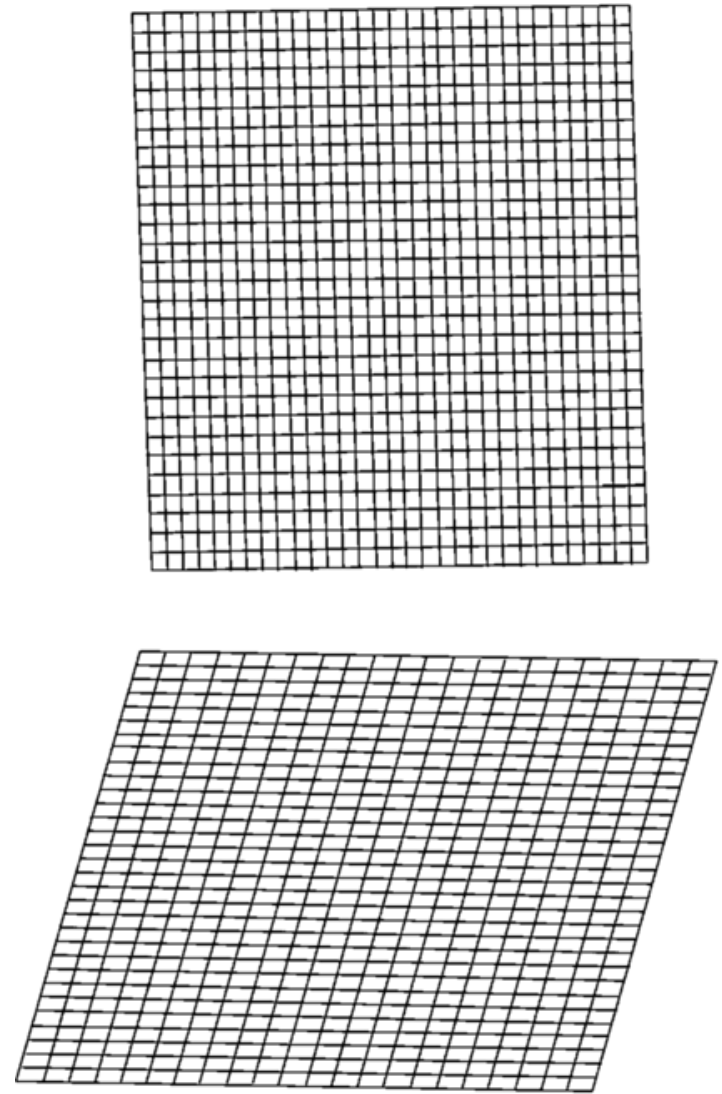

Figure 15. The uniform affine component of thin plate spline analyses on the means plotted in Figures 14 and 16. The top panel shows the results for means plotted in Figure 14. The bottom panel shows the results for the means plotted in Figure 16. As in Figure 13, the analysis was performed in the reverse direction.

shapes. The mean shape result has also be found to vary between studies. For instance, on average, shape was expanded in depth in Bingham et al. (2000) and also in Todd and Norman (1991). On the other hand, shape was compressed in Bingham (2003). However, a given participant does tend to be consistent in either expanding or compressing shapes from trial to trial and from one occasion to the next. Understanding of this consistency lies beyond the affine analysis (Bingham et al., 2000; Lind \& Börjesson, 2001; Todd \& Perotti, 1999).

In any case, the affine analysis predicts that the scaling of perceived distance and the scaling of shape should be independent, because the affine analysis is consistent with a scaled orthographic model of the optical information for object perception. Shape could easily be expanded while egocentric distance is compressed, or shape and distance could both be compressed, but by different amounts. An affine analysis also predicts that distance perception could be rendered relatively accurate by feedback information and calibration, whereas shape perception could remain distorted, unaffected by calibration of distance. This is exactly the pattern of results obtained in Bingham et al. (2000), Bingham, Bradley, et al. (2001), and Bingham (2003), as well as in the present experiments. Similarly, Crowell, Todd, and Bingham $(2000,2001)$ found that perception of egocentric distance and of exocentric distance could be independently recalibrated by distorted feedback, changing one without affecting the other.

Finally, the present experiments demonstrate that distortions in the perception of object distances and shapes cannot be captured by a single continuous one-to-one transformation of the underlying reach space. Certainly, a single uniform affine transformation of the space is not enough. We consistently found nonaffine or nonuniform components in transformation of the space. Often, we
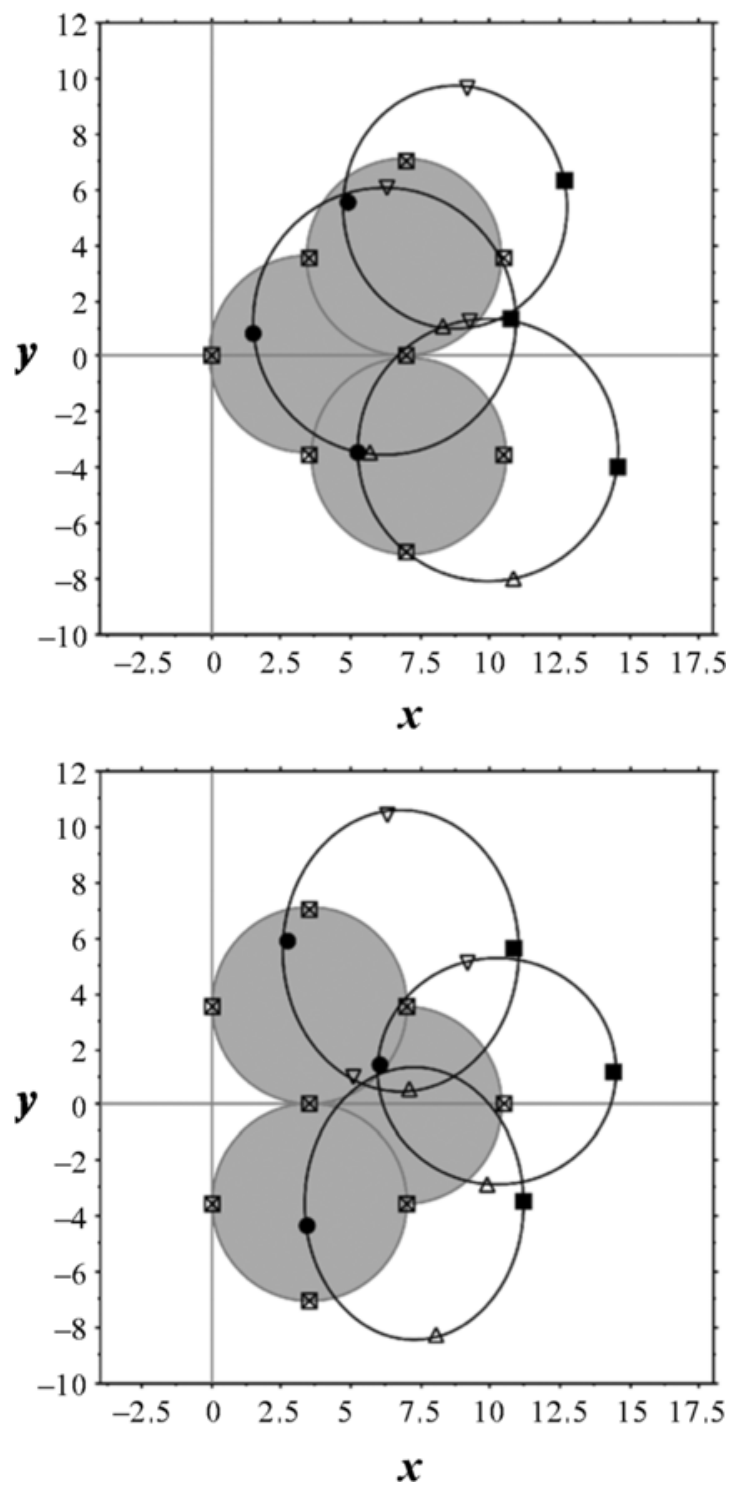

Figure 16. The same plot as in Figure 14 but for the 3 participants who did not yield significant differences between front and side points. 
also found uniform affine components, except in the case of the calibrated participants using dynamic binocular vision. Most often, the uniform affine component was compression in depth, and this is consistent with many other findings of slopes less than 1 in judged distance functions. Even a single nonaffine function, however, would fail to account for our results. Instead, we found that the space is folded, with a resulting loss of points, and torn, with a resulting gain of points. The clear implication is that more than one transformation was at work. Distortions in perceived distance (or position) are separate from distortions in perceived shape. There is no single coherent visual space, as now has been shown by this and related studies on egocentric and exocentric distance perception (Crowell et al., 2000, 2001; Loomis, Philbeck, \& Zahorik, 2002) and on size and distance or position perception (Gillam \& Chambers, 1985; Gruber, 1954; Kilpatrick \& Ittleson, 1953; see Gillam, 1995, and Sedgwick, 1986, 2001, for reviews.)

\section{REFERENCES}

BAIRD, J. C., \& BIERSDORF, W. R. (1967). Quantitative functions for size and distance judgments. Perception \& Psychophysics, 2, 161-166.

BINGHAM, G. P. (2003). Calibration of distance and size does not calibrate shape information: Comparison of dynamic monocular and static and dynamic binocular vision. Manuscript submitted for publication.

Bingham, G. P., Bradley, A., Bailey, M., \& Vinner, R. (2001). Accommodation, occlusion and disparity matching are used to guide reaching: A comparison of actual versus virtual environments. Journal of Experimental Psychology: Human Perception \& Performance, 27, 1314-1344.

Bingham, G. P., Crowell, J. A., \& TodD, J. T. (2001, May). Distortions of distance and shape do not reflect a single continuous transformation on reach space. Paper presented at the annual meeting of the Vision Sciences Society, Sarasota, FL.

Bingham, G. P., ZaAl, F., Robin, D., \& Shull, J. A. (2000). Distortions in definite distance and shape perception as measured by reaching without and with haptic feedback. Journal of Experimental Psychology: Human Perception \& Performance, 26, 1436-1460.

BRENNER, E., \& VAN DAMME, W. J. M. (1997). The distance used for scaling disparities is the same as the one used for scaling retinal size. Vision Research, 37, 757-764.

Brenner, E., \& Van Damme, W. J. M. (1999). Perceived distance, shape and size. Vision Research, 39, 975-986.

Crowell, J. A., Todd, J. T., \& Bingham, G. P. (2000, November). Distinct visuomotor transformations for visually guided reaching. Paper presented at the 41st Annual Meeting of the Psychonomic Society, New Orleans.

Crowell, J. A., Todd, J. T., \& Bingham, G. P. (2001, May). Distinct perceptual representations for visually guided reaches. Paper presented at the annual meeting of the Vision Sciences Society, Sarasota, FL.

Domini, F., \& CAUDE K, C. (1999). Perceiving surface slant from deformation of optic flow. Journal of Experimental Psychology: Human Perception \& Performance, 25, 426-444.

DRYDEN, I. L., \& MARDIA, K. V. (1998). Statistical shape analysis. Chichester, UK: Wiley.

FERRIS, S. H. (1972). Motion parallax and absolute distance. Journal of Experimental Psychology: Human Perception \& Performance, 95, 258-263.

Gilinsky, A. (1951). Perceived size and distance in visual space. Psychological Review, 58, 460-482.

GiLlam, B. (1995). The perception of spatial layout from static optical information. In W. Epstein \& S. J. Rogers (Eds.), Perception of space and motion (pp. 23-67). San Diego: Academic Press.

Gillam, B., \& ChAmbers, D. (1985). Size and position are incongruous:
Measurements on the Müller-Lyer figure. Perception \& Psychophysics, 37, 549-556.

GogeL, W. C. (1977). The metric of visual space. In W. Epstein (Ed.), Stability and constancy in visual perception: Mechanisms and processes (pp. 129-181). New York: Wiley.

GRUBER, H. (1954). The relation of perceived size to perceived distance. American Journal of Psychology, 67, 411-426.

Helmholtz, H. von (1925). Physiological optics (Vol. 3). New York: Optical Society of America.

HochberG, J. E. (1978). Perception (2nd ed.). Englewood Cliffs, NJ: Prentice-Hall.

HOWARD, I. P., \& ROGERS, B. J. (1995). Binocularvision and stereopsis. New York: Oxford University Press.

INDOw, T. (1991). A critical review of Luneburg's model with regard to global structure of visual space. Psychological Review, 98, 430453.

Johnston, E. B. (1991). Systematic distortions of shape from stereopsis. Vision Research, 31, 1351-1360.

Kilpatrick, F. P., \& ItTLESON, W. (1953). The size-distance invariance hypothesis. Psychological Review, 60, 223-231.

KoEnderink, J. J., \& VAN DOORN, A. J. (1987). Facts on optic flow. Biological Cybernetics, 56, 247-254.

KoENDERINK, J. J., \& VAN DOORN, A. J. (1991). Affine structure from motion. Journal of the Optical Society of America A, 8, 377-385.

LIND, M. (1996). Perceiving motion and rigid structure from optic flow: A combined weak-perspective and polar-perspective approach. Perception \& Psychophysics, 58, 1085-1102.

LIND, M., \& BÖRJESSON, E. (2001). Perceived structure from optic flow: Consistent versus variable mapping of 3-D Euclidean structure. Scandinavian Journal of Psychology, 42, 105-112.

LoOMIs, J. M., PhILBECK, J. W., \& ZAHORIK, P. (2002). Dissociation between location and shape in visual space. Journal of Experimental Psychology: Human Perception \& Performance, 28, 1202-1212.

LuNEBURG, R. K. (1950). The metric of binocular space. Journal of the Optical Society of America, 40, 627-642.

Norman, J. F. (2002). Two visual systems and two theories of perception: An attempt to reconcile the constructivist and ecological approaches. Behavioral \& Brain Sciences, 25, 73-96.

Norman, J. F., \& TODD, J. T. (1993). The perceptual analysis of structure from motion for rotating objects undergoing affine stretching transformations. Perception \& Psychophysics, 3, 279-291.

Pagano, C. C., \& Bingham, G. P. (1998). Comparing measures of monocular distance perception: Verbal and reaching errors are not correlated. Journal of Experimental Psychology: Human Perception \& Performance, 24, 1037-1051.

SEDGWICK, H. A. (1986). Space perception. In K. R. Boff, L. Kaufman, \& J. P. Thomas (Eds.), Handbook of perception and human performance (Vol. 1, pp. 21.1-21.57). New York: Wiley.

SEDGWICK, H. A. (2001). Visual space perception. In E. B. Goldstein (Ed.), Blackwell handbook of perception (pp. 128-167). Oxford: Blackwell.

Thompson, D. W. (1961). On growth and form. Cambridge: Cambridge University Press.

Tittle, J. S., Todd, J. T., Perotti, V. J., \& Norman, J. F. (1995). Systematic distortion of perceived three-dimensional structure from motion and binocular stereopsis. Journal of Experimental Psychology: Human Perception \& Performance, 21, 663-678.

TODD, J. T., \& BRESSAN, P. (1990). The perception of 3-dimensional affine structure from minimal apparent motion sequences. Perception \& Psychophysics, 48, 419-430.

TODD, J. T., \& NORMAN, J. F. (1991). The visual perception of smoothly curved surfaces from minimal apparent motion sequences. Perception \& Psychophysics, 50, 509-523.

Todd, J. T., \& Perotti, V. J. (1999). The visual perception of surface orientation from optical motion. Perception \& Psychophysics, 61, 1577-1589.

Todd, J. T., Tittle, J. S., \& Norman, J. F. (1995). Distortions of threedimensional space in the perceptual analysis of motion and stereo. Perception, 24, 75-86.

Wagner, M. (1985). The metric of visual space. Perception \& Psychophysics, 38, 483-495. 


\section{NOTES}

1. In the feedback conditions in which the participants could see both the stylus and the target, we wanted them to be able to see both the top and the bottom of the stylus when it was positioned behind the target. We recorded positions of both the top and the bottom of the stylus and computed the mean $x$ and $y$ coordinates to yield the location of the midpoint. We computed the absolute difference of the top and bottom $x$ coordinates in the monocular no-feedback condition, to evaluate how much the stylus varied from a vertical orientation. The mean orientation error was .08 rads $(S D=.06$ rads). If the participants misgauged the midpoint of the stylus by $\pm 3 \mathrm{~cm}$, this would have involved a mean measurement error of only $2.4 \mathrm{~mm}(S D=1.8 \mathrm{~mm})$.

2. Morphometrika is a Macintosh PowerPC program written by Jeffrey A. Walker, Department of Biology, University of Southern Maine, 96 Falmouth St., Portland, ME 04103 (e-mail:walker@usm.maine.edu). It is available on the Web at www.usm.maine.edu/ walker/software.html.

3. It is important to understand that the results of the TPS analysis do describe the changes in the space that occur as a result of the respective transformations - that is, the graphs shown in this article do represent the average distortions that were recorded in these experiments. Information about some aspects of the resulting changes to the space can be found in these results as demonstrated. However, the average changes represented by these graphs were produced by more than one transformation (e.g., compressing distances and expanding shapes), and thus, they represent only the results of the combined transformations. The TPS analysis does partition the total transformation into affine global (or uniform) and local (or nonuniform and nonaffine) components. If the perceptual transformation of position is global and affine (which it may be), whereas the perceptual transformation of shape is local (which is also possible), this analysis would also represent the respective positionand shape-specific transformations. However, we do not yet know the specif ic character of the respective transformations, and so we can make no conclusions in this regard. Nevertheless, we do describe similarities to the results of analyses of distance perception shown in another report and note these similarities as suggestive.

(Manuscript received September 3, 2002; revision accepted for publication July 10, 2003.) 